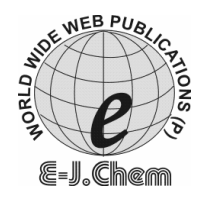

http://www.e-journals.net
ISSN: 0973-4945; CODEN ECJHAO

E-Journal of Chemistry 2009, 6(1), 39-46

\title{
Design of New Electrode Interface to Improve Transport of Atmospheric Pressure Ions into a Mass Spectrometer
}

\author{
FRANCIS BEAUDRY* and ROBERT B. MOORE \\ *Department of Veterinary Medicine, Faculty of Veterinary Medicine, \\ Groupe de Recherche en Pharmacologie Animal du Québec (GREPAQ) \\ University of Montréal, St-Hyacinthe, Québec, Canada. \\ Department of Physics, McGill University, Montreal, Canada. \\ francis.beaudry@umontreal.ca
}

Received 7 May 2008; Accepted 5 June 2008

\begin{abstract}
An intermediate electrode was developed to improve the transfer of ions in atmospheric pressure from a first location, the ion source, to a second location, the mass spectrometer. The new apparatus increase the efficiency of mass analysis of molecular constituents of liquids, including trace analysis of chemical entities, in which an electrospray (ES) or IonSpray ${ }^{\mathrm{TM}}$ (IS) technique is used to produce electrically charged droplets which divide and evaporate to form gaseous ions of the molecular constituents. The gas phase ions are transported to the mass spectrometer by an electric field generated by a new electrode design that separates the two fundamental functions of an electrospray or an IonSpray ${ }^{\mathrm{TM}}$, which are the nebulization of charged droplets and the transport of ions into the mass analyzer. The results suggest that the new apparatus provide a gain in signal intensity up to 10 compared with the commercial product. A significant improvement in ion transport results in higher precision and accuracy and/or reduction of the amount of material needed for analysis.
\end{abstract}

Keywords: Electrospray, IonSpray, Transport of atmospheric pressure, Mass spectrometer

\section{Introduction}

An atmospheric pressure ion (API) source is necessary when any instrument that separates molecules in a liquid phase is coupled to a detector that identifies the ions by manipulations in the gas phase. A common example is the use of High Performance Liquid Chromatography (HPLC) that separates pharmaceutical products coupled to a mass spectrometry (MS) in order to analyze the separated molecules. 
The analysis of ions by mass spectrometry is important since it enables the determination of chemical structures and concentrations of molecules in complex mixtures. However, all mass spectrometers operate by circulating ions in a high vacuum $\left(<10^{-5}\right.$ torr). It is therefore necessary to render the molecules in the liquid phase from HPLC into ions in the gas phase that are then transported into the vacuum of the spectrometer. This is accomplished by the API source. Figure 1 is a schematic of the role of an atmospheric ion source, coupling HPLC to mass spectrometry.

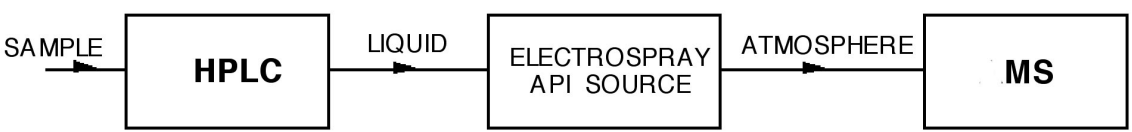

Figure 1. Schematic analysis process.

The most common API sources used to couple HPLC to MS are electrospray ${ }^{1,2}$ and IonSpray ${ }^{\mathrm{TM}}$ (IS) ${ }^{3}$. With these sources, the liquid from the HPLC is directed through the free end of a capillary typically set at 3 to $5 \mathrm{kV}$. In the case of pure electrospray, the high electric field at the tip of the capillary pulls the liquid emanating from the capillary into a fine jet that breaks up, typically a millimeter from the tip of a 100 micron capillary, into a fine spray of electrified droplets. In the case of IonSpray ${ }^{\mathrm{TM}}$ this breakup into fine drops is assisted by the flow of high pressure nitrogen over the capillary tip so that more liquid can be accommodated. The fine droplets in the spray evaporates in about one millisecond to liberate charged molecules from the droplets as ions ${ }^{4,5}$, which the electric field of the capillary tip then forces toward the entrance of the mass spectrometer. Figure 2 shows schematically how an electric field is commonly used to accomplish these functions.

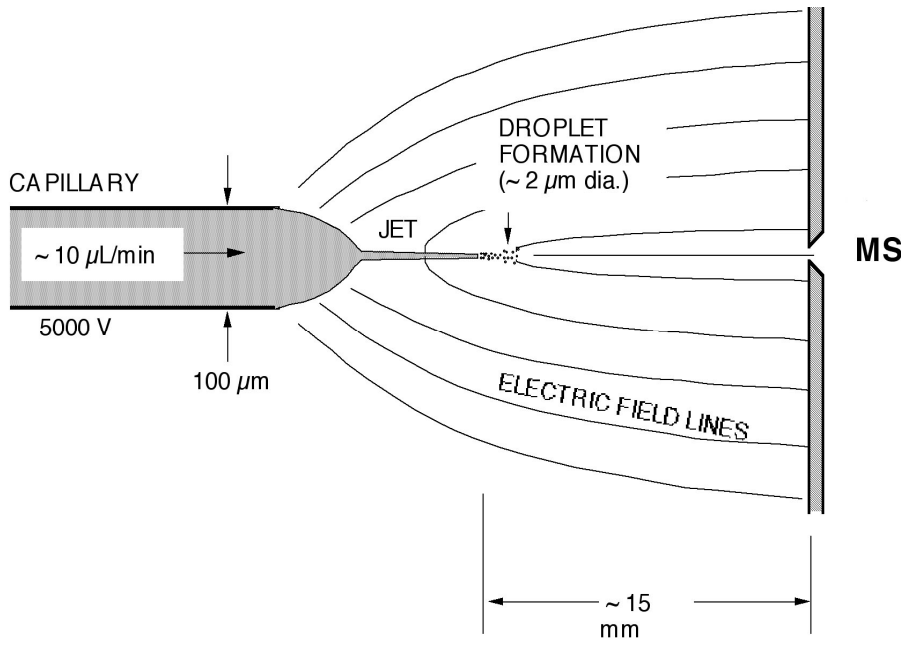

Figure 2. A typical electrospray source.

Very early during the implementation of electrospray with mass spectrometry, it was noticed that ion collection efficiency was poor. In order to improve gas phase ion generation efficiency, the electrospray source was modified to assist electric nebulization by highpressure gas swept over the electrode tip, typically at speeds of up to $200 \mathrm{~m} / \mathrm{s}$. This improved interface proposed by Bruins et $a l .{ }^{3}$ is currently the most widely used version of electrospray interface in LC-MS. The modified source can accommodate up to ten times 
more liquid compared to conventional electrspray. However it is only a small fraction of the liquid that is actually evaporated without heat assistance. Furthermore, the atmosphere in which the ions are produced, presumably near the electrode tip, is under rapid flow $(<100$ $\mathrm{m} / \mathrm{s}$ ), making extraction of these ions for delivery toward the MS entrance very difficult. Consequently, only a small fraction of the solute material ends up being transported as ions into the MS. Moreover, the ions tend to follow the electric field lines and these diverge from the needle so that most of them fall on the interface plate of the mass spectrometer away from the orifice leading into the mass spectrometer ${ }^{6}$. A large number of the ions are therefore lost. Larger orifices leading into the vacuum could be used to pull in ions from a larger region but this would be at the expense of considerably enlarging the pumping system required to maintain an adequate vacuum in the mass spectrometer and a higher risk of system failure. Clearly, improvement to the electrospray interface would lead to considerable enhancement of LC-ESI/MS and this has motivated our involvement in this research field ${ }^{8-10}$. An ion funnel interface was suggested ${ }^{9}$ but the implementation of such design is complex and not practical.

Our primary objective was to develop a method and/or device which allowed a more efficient transfer of ions at atmospheric pressure from the ion source to the mass spectrometer. The new apparatus developed would increase the efficiency of analysis, in which an electrospray technique is used to produce electrically charged droplets and evaporate them to form gas phase ions of the molecular constituents. The gas phase ions are transported to the mass spectrometer by an electric field generated by a new electrode design that separates the two fundamental functions of an electrospray, which are the formation of gas phase ions and transport of the ions into the mass spectrometer. Significant improvement in ion transport can potentially result in higher precision and accuracy and reduce the amount of sample needed for bioanalytical methodologies.

\section{Design of the new aperture}

The tested aperture propose the use of an intermediate electrode with a high electric field, in addition to the ion production field, to direct the ions from an API source into an orifice leading into a mass spectrometer. Essentially, this divides the operation of the API source into two distinct functions; the creation of the ions and their delivery into the orifice ${ }^{7}$. In present electrospray ion sources, the electric field surrounding the capillary tip does both. However, the electric field that is necessary for the production of the ions (i.e. a field which is very high at the capillary tip) is very inefficient for the delivery of these ions to the distant orifice where the electric field is much weaker because of the divergence of the field lines. Therefore, in our suggested design as soon as the ions are liberated from the electrospray, the creation field is essentially replaced by a drift field designed for more efficient ion transport. It is done by introducing an intermediate electrode specifically for this purpose. In essence, as soon as the ions are liberated from the electrospray, the creation field is replaced by a drift field designed for efficient ion transport ${ }^{8-10}$. Thus rather than trying to refocus the electric fields from the capillary tip toward the orifice, a new field is created on the downstream side of the electrode that is designed from its start to focus on the orifice. Figure 4 shows the implementation of the concept of this new electrode to a typical mass spectrometer used with an Ionspray ${ }^{\mathrm{TM}}$ source, where the rendering of the liquid into a spray is enhanced by the flow of high-pressure nitrogen over the capillary tip. The Figure 4 shows the implementation of the concept of the current design to a typical mass spectrometer used with an Ionspray $^{\mathrm{TM}}$ source (1), where the rendering of the liquid into a spray is enhanced 
spray is enhanced by the flow of high-pressure nitrogen (2) over the capillary tip. Such a source is typically directed away from the entrance of the mass spectrometer so that the large background droplets that do not evaporate are not blown into the entrance by the gas flow. In addition, to further reduce the entry of liquid into the vacuum of the spectrometer, a reverse flow of nitrogen gas (3) is directed away from the spectrometer entrance (4) so as to sweep away these drops. Because of this, the entrance geometry is a reversed cone as shown in the Figure 4. In this case the electrode (5) that produces the drift field must be shaped as shown. Although in this configuration the field lines inherently diverge from the axis more than in the planar case, the addition of the electrode still results in a substantial increase in the electric field at the spectrometer entrance, and hence in the delivery of ions to the entrance. A further advantage of the increase drift field is a reduction in the drift time of the ions into the mass spectrometer, which reduces interactions of the ions with atmospheric gas and solvent molecules that can lead to charge loss and/or fragmentation.

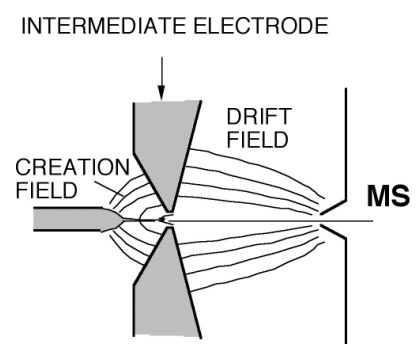

Figure 3. Basic Design using an Intermediate Electrode.

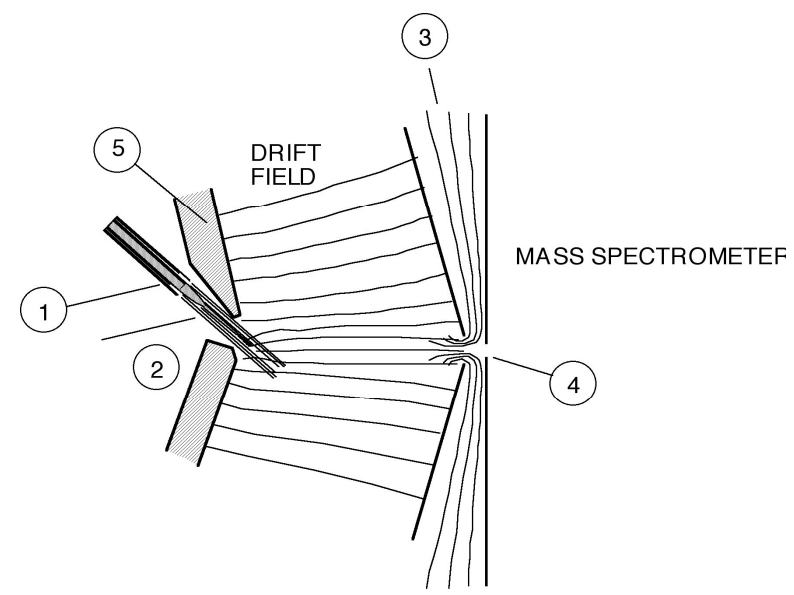

Figure 4. Configuration implemented with an Ion Sray ${ }^{\mathrm{TM}}$ source and an API 3 plus mass spectrometer from AB-MDS Sciex.

\section{Experimental}

\section{Materials and reagents}

Midazolam and terconazole were purchased from Sigma (St-Louis, MO, USA). Ammonium acetate, and methanol were obtained from Fisher (Fair Lawn, NJ, USA). The transport electrode was custom made with an alloy of cadnium-aluminium and introduced on the IonSpray $^{\mathrm{TM}}$ ion source as shown in Figure 5. 


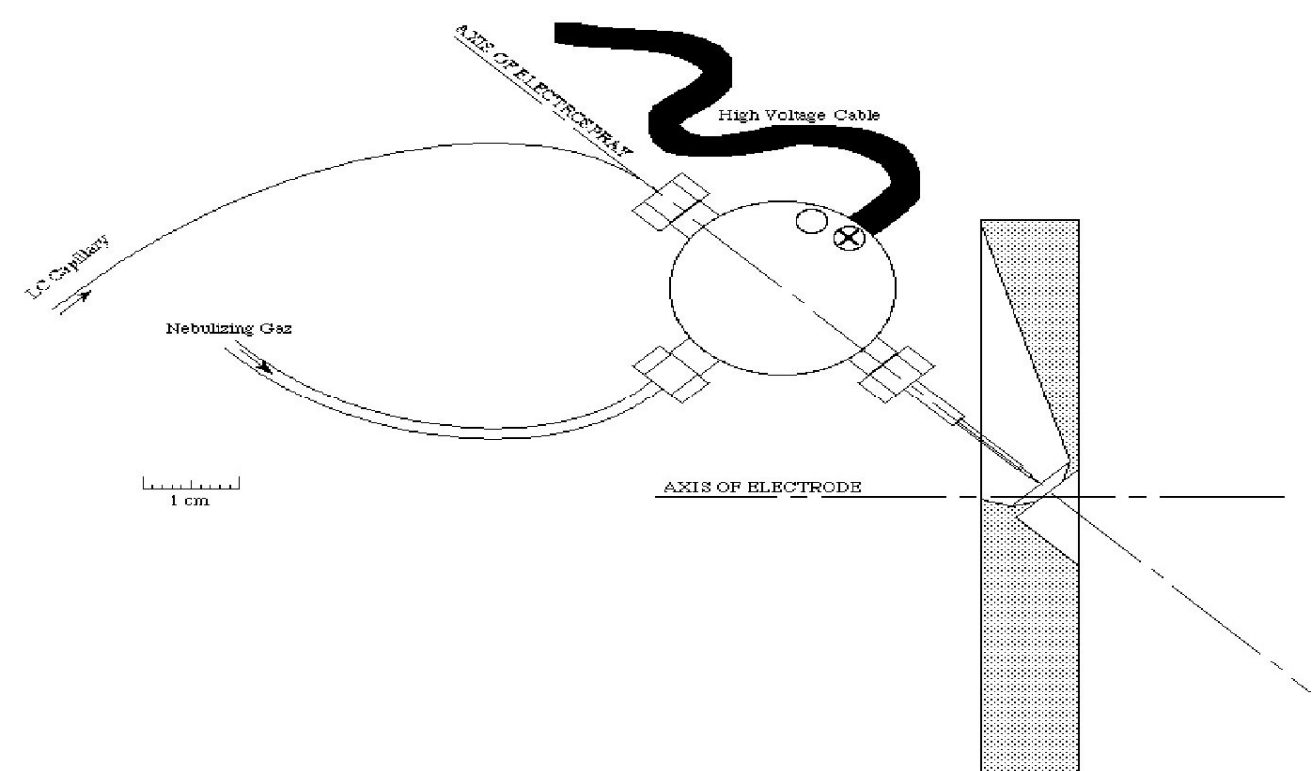

Figure 5. Implementation of the intermediate electrode with the IonSpray ${ }^{\mathrm{TM}}$ source.

\section{Instrumentation}

The HPLC system consisted of a Hewlet-Packard 1090 pump (Palo Alto, CA, USA). The LC/MS/MS system used was a PESCIEX API 3 plus (Foster City, CA, USA) equipped with an IonSpray ${ }^{\mathrm{TM}}$ source and infusion experiments were performed with a single-syringe infusion Pump from Kd Scientific ( New Hope, PA, USA).

\section{Flow Injection and infusion experiments}

Five $\mu \mathrm{L}$ aliquot of a $10 \mathrm{pg} / \mu \mathrm{L}$ solutions (compounds dissolved in methanol) were injected into the mass spectrometer. The mobile phase was composed of methanol $(\mathrm{MeOH})$ and 25 $\mathrm{mM}$ ammonium acetate in water (80:20) set at a flow rate of $10 \mu \mathrm{L}$ per minute. For the infusion experiments, the compounds were dissolved $(10 \mathrm{pg} / \mu \mathrm{L})$ in 80:20 methanol:25 mM ammonium acetate in water and the infusion rate was $10 \mu \mathrm{L}$ per minutes. Other typical mobile phases used in LC/MS have been tested with comparable results. The analytes were monitored using a PESCIEX API 3 plus LC/MS/MS system equipped with an IonSpray ${ }^{\mathrm{TM}}$ ion source (modified and original version), with the capillary electrode set at $5000 \mathrm{~V}$ and the nitrogen pressure of 30 PSI. The monitoring was performed in full scan at a rate of 0.5 scan per second as well as in selected ion monitoring mode (SIM) with a dwell time of $100 \mathrm{~ms}$

\section{Results and Discussion}

The configuration shown in Figure 4 has been implemented with an Ion Spray ${ }^{\mathrm{TM}}$ source and an API 3 plus mass spectrometer from AB-MDS Sciex (Concord, Ontario). Tests have been carried out under a variety of conditions and with a variety of solutes and solvents. Results with midazolam, terconazole and insulin using a mobile phase composed of $80 \%$ methanol and $20 \% 25 \mathrm{mM}$ ammonium acetate in water with a flow rate from the capillary of $10 \mu \mathrm{L} / \mathrm{min}$ suggest an increase of 2 to 10 fold in signal intensity. Figure 6 shows representative results with midazolam using the intermediate electrode. Almost a 10-fold increase in signal 
intensities has been observed using a voltage of $3000 \mathrm{~V}$ on the intermediate electrode. Figure 7 shows a 3 -fold increase in signal intensities for terconazole using identical LC conditions. In these cases, it was observed that following 3000 to 3500 volts applied on the drift electrode, the highest that could be achieved in the system that was tested, the delivery of the ions into the spectrometer was enhanced by about a factor of 3 to 10. Figure 7 shows the necessity of a high electric field given that a potential of 2500 volts resulted in a 3 time increase in analyte response. On other tests, enhancements reached a factor of 20 but were not reproducible.

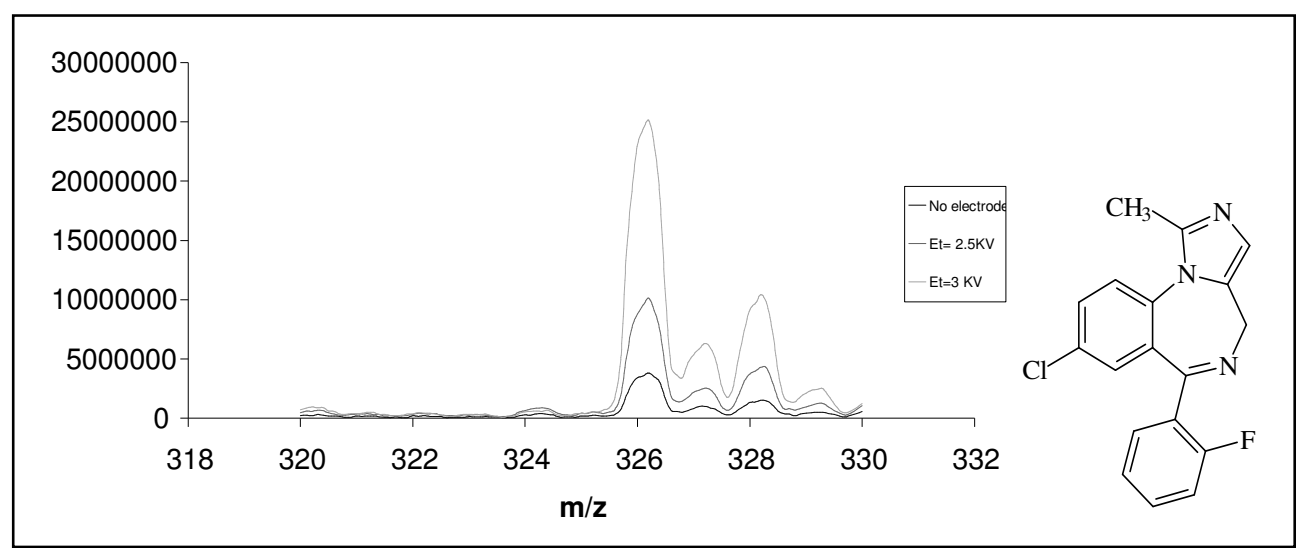

Figure 6. Midazolam results.

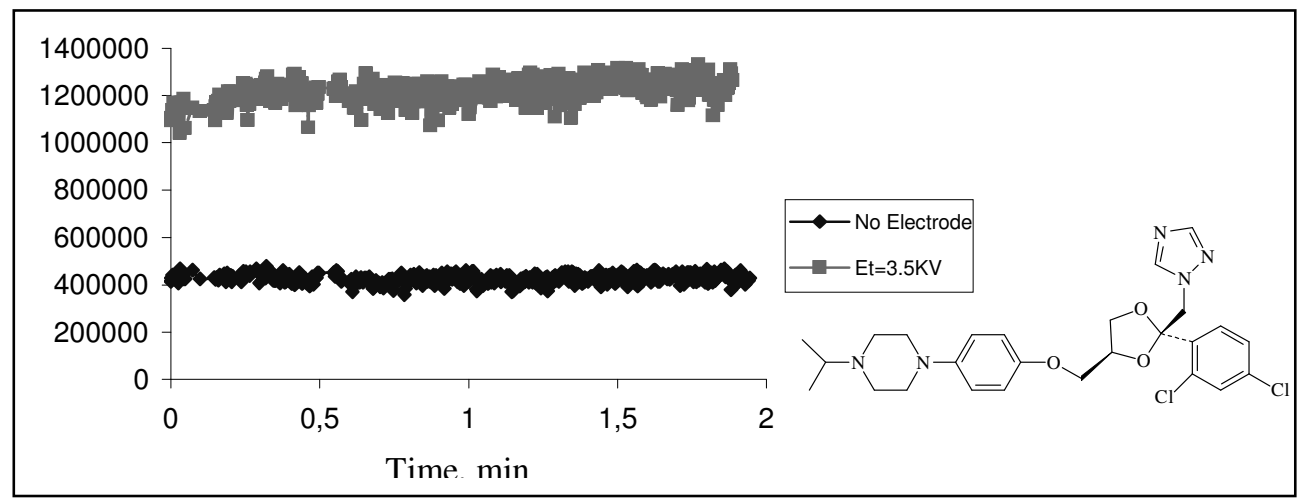

Figure 7. Terconazole results.

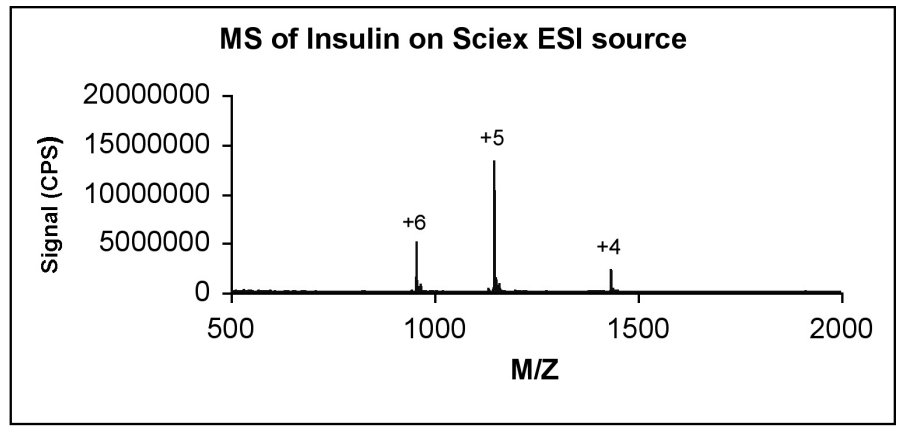




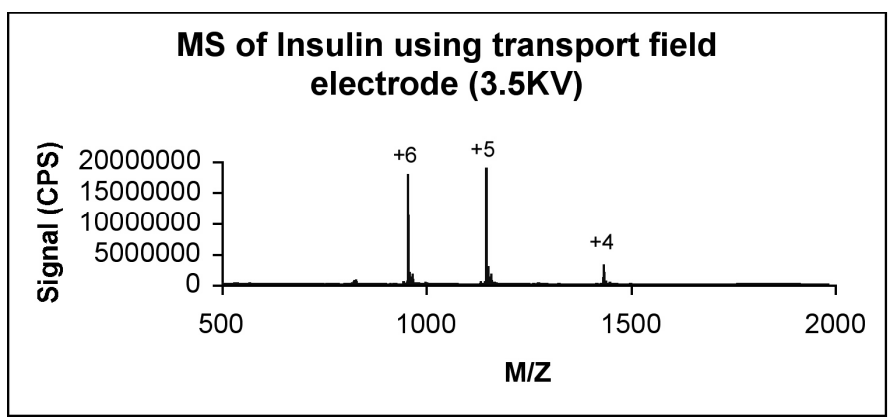

Figure 8. Insulin results.

Results with insulin shown in Figure 8, suggested that the effect of the transport field is proportional to the charge states of the ion, which could be interpreted as an indirect demonstration of the separation of both fundamental functions of the electrospray or Ion Spray ${ }^{\mathrm{TM}}$ source using the current aperture $(\vec{F}=q \vec{E})$.

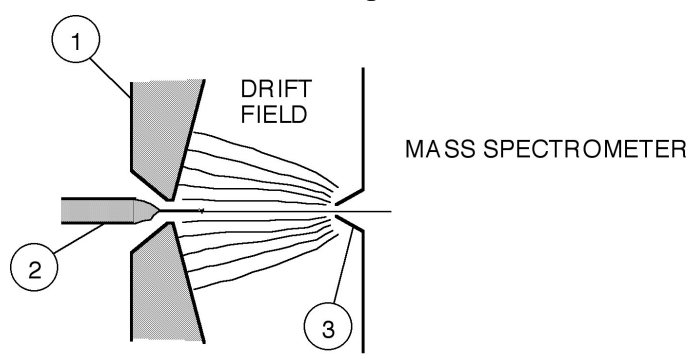

Figure 9. Concept design, version \#1.

In order to improve and adapt the current design to other mass spectrometer, similar concept designs should be investigated. Figure 9 shows an implementation of a drift electrode (1) where the geometry of the entrance of the mass spectrometer (3) has been modified to concentrate the electric field lines from the drift electrode onto the spectrometer entrance. This should result in a further improvement in the delivery of the ions from the source (2) into the spectrometer. Figure 10 shows an implementation of a drift electrode (1) designed to separate the electric field in which the ions are produced (2) from the drift field that directs them to the mass spectrometer (3). In this way, by proper design, the ion creation field and the ion drift field can be separately optimized, thereby further enhancing the delivery of ions to the mass spectrometer from a given amount of sample.

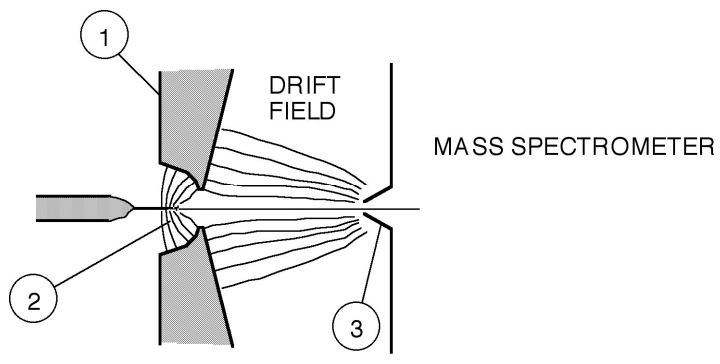

Figure 10. Concept design, version \#2. 


\section{Conclusion}

Experiments have been carried out under a variety of conditions and with a variety of solutes and solvents and we have observed an increase of 2 to 10 fold in signal intensity when the ion drift field is separated from the ion creation field. The new electrode greatly increases the electric field that drifts ions from an atmospheric pressure ion source toward the orifice leading to the mass spectrometer. The electrode design combined with modifications to the entrance geometry to help focus the drift field lines into the orifice leading to a mass spectrometer has the potential to enhance the ion collection rate by having geometry near the exit of the liquid from a capillary that is specifically designed to enhanced ion production and transport. The implementation of an intermediate electrode designed to achieve this separation significantly increases the ion transport from atmospheric pressure into a mass spectrometer. In this way, using proper design, the ion creation field and the ion drift field can be separately optimized, thereby further enhancing the delivery of ions to the mass spectrometer from a given amount of sample.

\section{References}

1. Yamashita M and Fenn J B, J Phys Chem., 1984, 88, 4451-4459.

2. Aleksandrov M L, Gall L N, Krasnov N V, Nikolaev V I, Pavlenko V A and Shkurov V A, Doklady Akademii Nauk SSSR [ Phys. Chem.,] 1984, 277, 379-383.

3. Bruins A P, Covey T R and Henion J D, Anal Chem., 1987, 59, 2642-2646.

4. Iribarne J V and Thompson B A, J Chem and Phys., 1976, 64, 2287-2294.

5. Thompson B A and Iribarne J V, J Chem and Phys., 1979, 71, 4451-4463.

6. Busman M, Sunner J and Vogel C R, J Am Soc Mass Spectrom., 1991, 2, 1-10.

7. Kim T, Tolmachev A V, Harkewicz R, Prior D, Anderson G, Udseth H R, Smith R D, Bailey T H, Rakov S and Futrell J H, Anal Chem., 2000, 72, 2247-2255.

8. Tolmachev A V, Kim T, Udseth H R, Smith R D, Bailey T H and Futrell J H, Int J Mass Spectrom., 2000, 203, 31-47.

9. Shaffer S A, Prior D C, Anderson G A, Udseth H R and Smith R D, Anal Chem., 1998, 70, 4111-4119.

10. Anderson A, Prior D C, Udseth H R and Smith R D, Rapid Commun Mass Spectrom., 1997, 11, 1813-1817. 


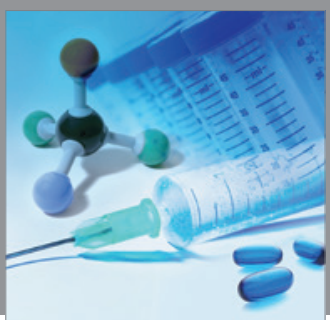

International Journal of

Medicinal Chemistry

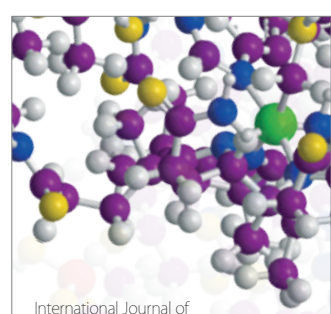

Carbohydrate Chemistry

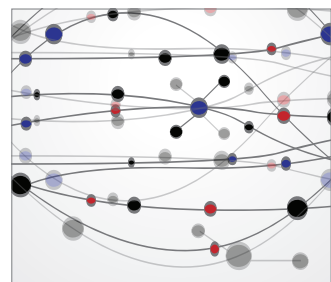

The Scientific World Journal
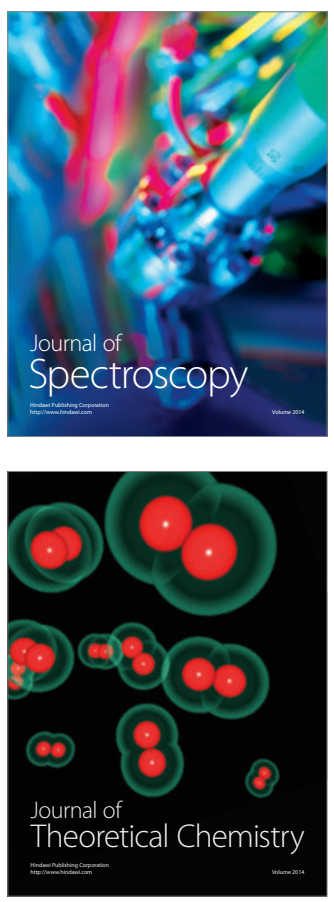
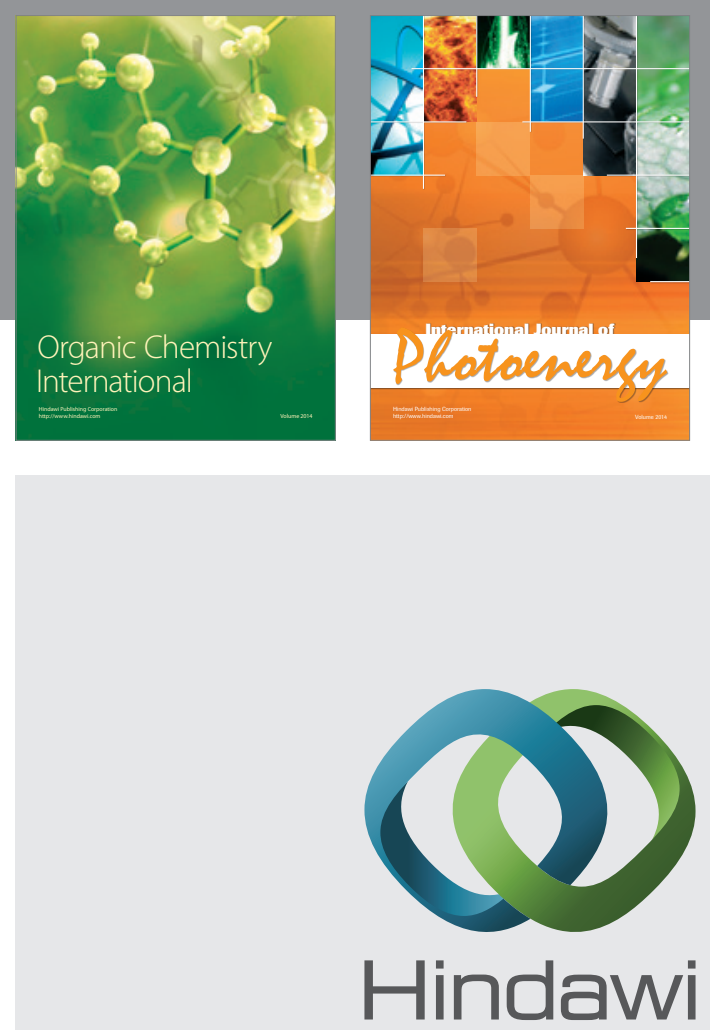

Submit your manuscripts at

http://www.hindawi.com
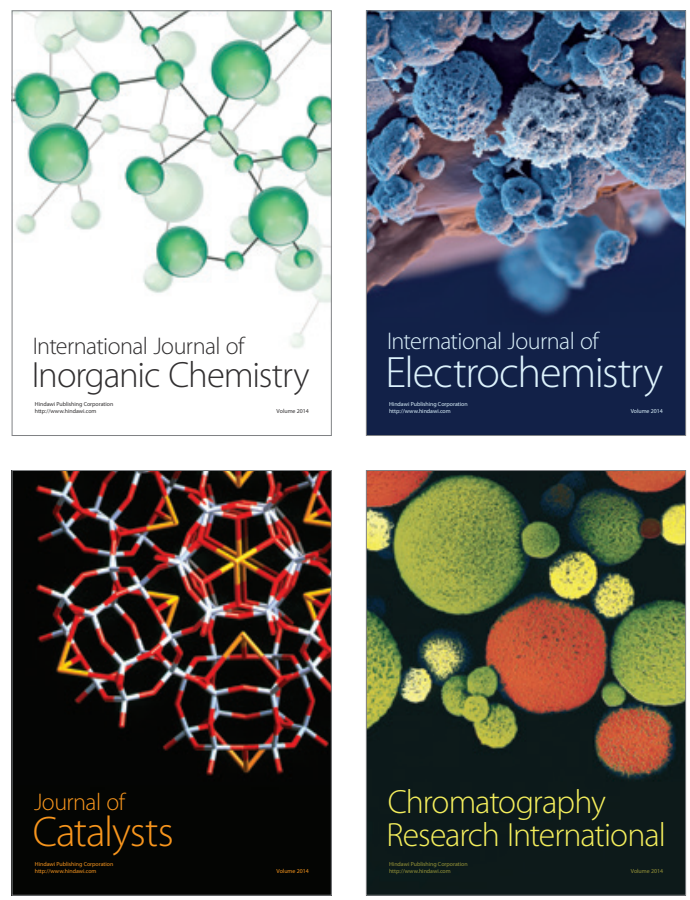
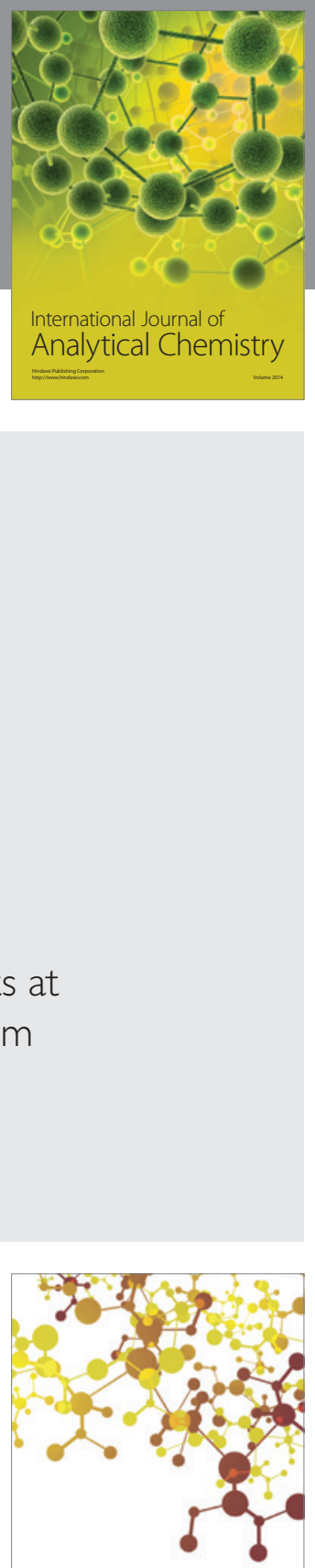

Journal of

Applied Chemistry
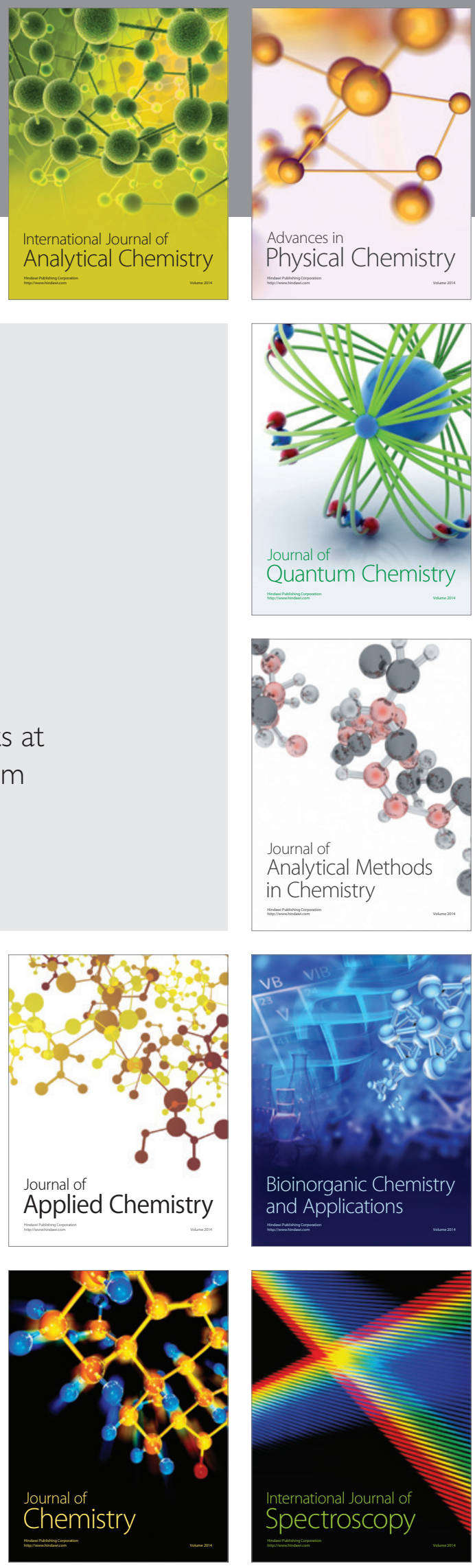